\title{
Avaliação do Equilíbrio Funcional e Qualidade de Vida em Pacientes com Espondilite Anquilosante
}

\section{Assessment of Functional Balance and Quality of life Among Patients with Ankylosing Spondylitis}

\author{
Marcelo Cardoso de Souza ${ }^{(1)}$, Gabriela da Costa Tutiya ${ }^{(1)}$, Anamaria Jones ${ }^{(1)}$, \\ Império Lombardi Júnior ${ }^{(2)}$, Jamil Natour ${ }^{(3)}$
}

\begin{abstract}
RESUMO
Objetivo: Avaliar o equilíbrio funcional e a qualidade de vida em pacientes com espondilite anquilosante. Tipo de estudo: Corte transversal. Métodos: Foram avaliados 60 indivíduos, sendo 30 com espondilite anquilosante (EA) e 30 saudáveis, por meio da versão brasileira da escala de equilíbrio de Berg, questionário SF-36 para avaliação da qualidade de vida e escala visual analógica da dor (EVA). Resultados: O escore da escala de Berg foi, respectivamente, para os grupos EA e controle de 48,83 e 55,5 , com diferença estatisticamente significante. Houve correlação positiva entre a escala de Berg e a de EVA $(r=0,469)$ e entre esta escala e o domínio referente à capacidade funcional do SF-36 $(r=0,648)$. A EVA teve correlação negativa com o SF-36 nos seguintes domínios: capacidade funcional $(r=-0,753)$, aspecto físico $(r=-0,612)$, estado geral de saúde $(r=-0,607)$, dor $(r=-0,827)$ e vitalidade $(r=-0,532)$. Conclusão: Os pacientes com EA possuem pior equilíbrio funcional, pior qualidade de vida e mais dor que indivíduos saudáveis. O pior equilíbrio correlacionou-se com pior capacidade funcional e dor.
\end{abstract}

Palavras-chave: espondilite anquilosante, equilíbrio, qualidade de vida.

\section{INTRODUÇÃO}

A espondilite anquilosante (EA) é uma doença inflamatória crônica que afeta primariamente o esqueleto axial. Além da articulação sacroilíaca e da coluna vertebral, as articulações periféricas podem estar acometidas em até 30\% dos casos, levando, assim, a comprometimento funcional importante ${ }^{(1-4)}$. As alterações estruturais na coluna vertebral começam precocemente e são $\operatorname{progressivas}^{(5)}$. Segundo

\begin{abstract}
Objective: To assess functional balance and quality of life among patients with ankylosing spondylitis. Type of study: Crosssectional. Methods: Sixty individuals were evaluated: 30 with ankylosing spondylitis (AS) and 30 healthy individuals - using the Brazilian version of the Berg Balance Scale, SF-36 questionnaire for the assessment of quality of life and a visual analogue scale (VAS) for pain. Results: The score of the scale of Berg was respectively for groups EA and control of 48.83 and 55.5 with statistically significant difference. There was a positive correlation between the Berg Scale and the VAS $(r=0,469)$ and between this scale and the functional capacity domain of the $S F-36(r=$ $0,648)$. There was a negative correlation between the VAS and following domains of the SF-36: functional capacity $(r=-0,753)$, physical aspects $(r=-0,612)$, overall health $(r=-0,607)$, pain $(r$ $=-0,827)$ and vitality $(r=-0,532)$. Conclusion: Patients with AS exhibit worse functional balance, a poorer quality of life and more pain than healthy individuals. Poorer balance was correlated to pain and worse functional capacity.
\end{abstract}

Keywords: ankylosing spondylitis, balance, quality of life.

Khan $^{(6)}$, o espondilítico adota postura de anteriorização da cabeça, retificação da lordose cervical e aumento da cifose dorsal, assumindo a chamada "postura do esquiador". De acordo com Ozgul et al.(4), a EA também afeta a qualidade de vida dos pacientes no âmbito pessoal, familiar e profissional.

Existem alguns instrumentos de avaliação de equilíbrio já bem descritos, como a plataforma de equilíbrio Biodex Stability System e magnetometria ${ }^{(5,7)}$. A escala de equilíbrio

Recebido em 8/1/2008. Aprovado, após revisão, em 9/5/2008. Os autores declaram nenhum conflito de interesse.

Setor de Coluna Vertebral, Procedimentos e Reabilitação em Reumatologia da Disciplina de Reumatologia da Universidade Federal de São Paulo/Escola Paulista de Medicina (Unifesp/EPM), SP, Brasil.

1. Fisioterapeutas da Disciplina de Reumatologia da Unifesp

2. Professor adjunto do Curso de Fisioterapia da Unifesp

3. Professor da Disciplina de Reumatologia da Unifesp.

Endereço para correspondência: Jamil Natour, Disciplina de Reumatologia, Unifesp/EPM, Rua Botucatu, 740, 04023-900, São Paulo, SP, e-mail: jnatour@unifesp.br 
de Berg é uma escala validada e traduzida para o português, que avalia o equilíbrio funcional estático e dinâmico. É ferramenta de fácil e rápida aplicação, e com baixo custo em relação aos equipamentos usados em outros estudos. A escala de Berg tem o objetivo de avaliar o equilíbrio funcional, por meio de quatro tarefas comuns que envolvem o equilíbrio estático e o dinâmico, como, alcançar, girar, transferir-se, permanecer em pé e levantar-se. A realização das tarefas é avaliada pela observação direta e a pontuação varia de 0 a 4 , totalizando o máximo de 56 pontos. Um escore abaixo de 45 indica equilíbrio pobre. O teste satisfaz várias necessidades, incluindo a descrição quantitativa do equilíbrio, o monitoramento do progresso clínico do paciente e a avaliação da eficácia da intervenção realizada na prática clínica e em estudos ${ }^{(8)}$. A escala apresenta sensibilidade que varia de $82 \%$ a $91 \%$ e especificidade que varia de $70 \%$ a $95 \%$, para avaliar risco de queda em idosos ${ }^{(9-11)}$. Este teste teve validade externa que se compara a outras escalas de equilíbrio e mobilidade, como Barthel Mobility Sub-Scale, Balance Sub-Scale e Timed Up o Go Test ${ }^{(12)}$.

Levando-se em consideração as alterações biomecânicas que podem conduzir ao comprometimento do equilíbrio em pacientes com EA, o objetivo primário deste estudo foi avaliar o equilíbrio em pacientes com EA e, secundariamente, correlacionar o equilíbrio com dor e qualidade de vida.

\section{MÉTODOS}

Este foi um estudo tipo corte transversal, controlado, no qual foram avaliados o equilíbrio, a dor e a qualidade de vida em pacientes com EA. Os pacientes foram pareados por sexo e idade com indivíduos-controle.

Foram avaliados 60 indivíduos: $30 \mathrm{com}$ EA selecionados seqüencialmente no ambulatório da Disciplina de Reumatologia da Universidade Federal de São Paulo (Unifesp), 22 homens e oito mulheres; e 30 indivíduos-controle saudáveis, 21 homens e nove mulheres selecionados entre acompanhantes e funcionários do hospital.

O projeto foi aprovado pelo Comitê de Ética da Unifesp e todos os participantes assinaram o termo de consentimento livre e esclarecido.

Foram incluídos pacientes classificados como EA, segundo os Critérios de Nova Iorque Modificado de 1984(13), com idade entre 18 a 72 anos.

Foram excluídos pacientes com problemas cardiovasculares descompensados, doença neurológica ou psiquiátrica, amputação de membros inferiores, impossibilitados de permanecer em pé sozinhos, sem a ajuda de outra pessoa, deficiência visual ou auditiva grave que estivessem em uso de sedativos, portadores de diabetes melito e com prótese de quadris ou joelhos.

Os pacientes e os indivíduos-controle foram avaliados uma única vez pelo mesmo fisioterapeuta, familiarizado com os testes a serem aplicados, utilizando a versão brasileira da escala de equilíbrio de $\operatorname{Berg}^{(8)}$, a versão brasileira do SF-36 ${ }^{(14)}$ e a escala visual analógica de dor de 0 a $10 \mathrm{~cm}^{(15)}$.

\section{ANÁLISE ESTATÍSTICA}

As variáveis idade e domínio dor do SF-36 apresentaram distribuição normal pelo teste de Kolmogorov-Smirnov, logo os grupos foram comparados utilizando o teste $t$ de Student.

As variáveis Berg, EVA e demais componentes do SF-36 não possuíam distribuição normal pelo teste de Kolmogorov-Smirnov, logo os grupos foram comparados pelo teste de Mann-Whitney.

Para a variável sexo, foi utilizado o teste qui-quadrado.

As correlações de Spearmam foram utilizadas entre todas as variáveis avaliadas no grupo de EA.

\section{RESULTADOS}

Os pacientes com EA e os indivíduos-controle foram considerados homogêneos em relação a idade $(p=0,942)$ e sexo $(p=0,774)$.

A média de idade do grupo de pacientes foi de 45,6 anos e no grupo-controle foi de 45,3 anos, $(\mathrm{p}=0,942) \mathrm{e}$ a média do tempo de diagnóstico do grupo de pacientes com EA foi de 105,3 meses.

Em relação à pontuação da escala de equilíbrio de Berg e a escala visual analógica de dor, os dois grupos foram significativamente diferentes com $\mathrm{p}<0,001$ (Tabela 1 ).

\section{TABELA 1}

MÉdia E DESVIO-PADRÃO DAS VARIÁVEIS: IDADE, SEXO, ESCALA VISUAL ANALÓGICA DE DOR (EVA), ESCALA DE BERG E TEMPO DE DIAGNÓSTICO

\begin{tabular}{lccc}
\hline & Grupo EA & Grupo-controle & p \\
\hline Idade (anos) & $45,3 \pm 12,2$ & $45,6 \pm 12,6$ & 0,942 \\
Sexo (masculino/feminino) & $21 / 9$ & $22 / 8$ & 0,774 \\
EVA (cm) & $5,23 \pm 12,7$ & $1,16 \pm 1,36$ & $<0,001^{*}$ \\
Escala de Berg & $48,83 \pm 6,0$ & $55,5 \pm 0,93$ & $<0,001^{*}$ \\
Tempo diagnóstico (meses) & $105,3 \pm 89,4$ & & \\
\hline
\end{tabular}

* valor estatisticamente significativo. 
Os dados do SF-36 de ambos os grupos estão descritos na Tabela 2.

TABELA 2

AvaliaÇ̃̃o dos DOMÍNIOS DO SF-36 DEMONSTRAdOS EM MÉDIA E DESVIO-PADRÃO

\begin{tabular}{lccc}
\hline SF-36 & Grupo EA & Grupo-controle & $\mathrm{p}$ \\
\hline Capacidade funcional & $50,17 \pm 21,1$ & $92,00 \pm 9,7$ & $\mathrm{p}<0,001^{*}$ \\
\hline Aspectos físicos & $40,67 \pm 40,2$ & $92,50 \pm 19,8$ & $\mathrm{p}<0,001^{*}$ \\
\hline Dor & $43,77 \pm 21,0$ & $81,53 \pm 18,4$ & $\mathrm{p}<0,001^{*}$ \\
\hline Estado geral de saúde & $49,33 \pm 23,3$ & $82,50 \pm 13,6$ & $\mathrm{p}<0,001^{*}$ \\
\hline Vitalidade & $50,50 \pm 27,8$ & $77,60 \pm 14,3$ & $\mathrm{p}<0,001^{*}$ \\
Aspectos emocionais & $54,78 \pm 43,2$ & $85,00 \pm 26,1$ & $\mathrm{p}=0,004^{*}$ \\
Aspetos sociais & $65,98 \pm 32,0$ & $78,89 \pm 29,6$ & $\mathrm{p}=0,04$ \\
\hline Saúde mental & $64,00 \pm 23,2$ & $77,47 \pm 18,8$ & $\mathrm{p}=0,015$ \\
\hline
\end{tabular}

$\mathrm{EA}=$ espondilite anquilosante; $\mathrm{p}=$ valor da significância estatística; * valor estatisticamente significativo.

Houve correlação negativa entre a escala de Berg e a da EVA $(r=-0,469, p=0,009)$ (Figura 1). Entre a escala de Berg e o domínio referente à capacidade funcional do SF-36 houve correlação positiva, ou seja, quanto melhor o resultado da escala de Berg, melhor a capacidade funcional dos pacientes com EA $(r=0,648, p<0,001)$ (Figura 2).

O tempo de diagnóstico não apresentou correlação com as variáveis do SF-36, exceto com a capacidade funcional do SF-36 $(r=0,363$ e p = 0,049). Em relação à variável dor, avaliada pela EVA e pela escala de Berg, não houve correlação com o tempo de diagnóstico $(\mathrm{r}=-0,072 \mathrm{e}$ $\mathrm{r}=0,33$, respectivamente).

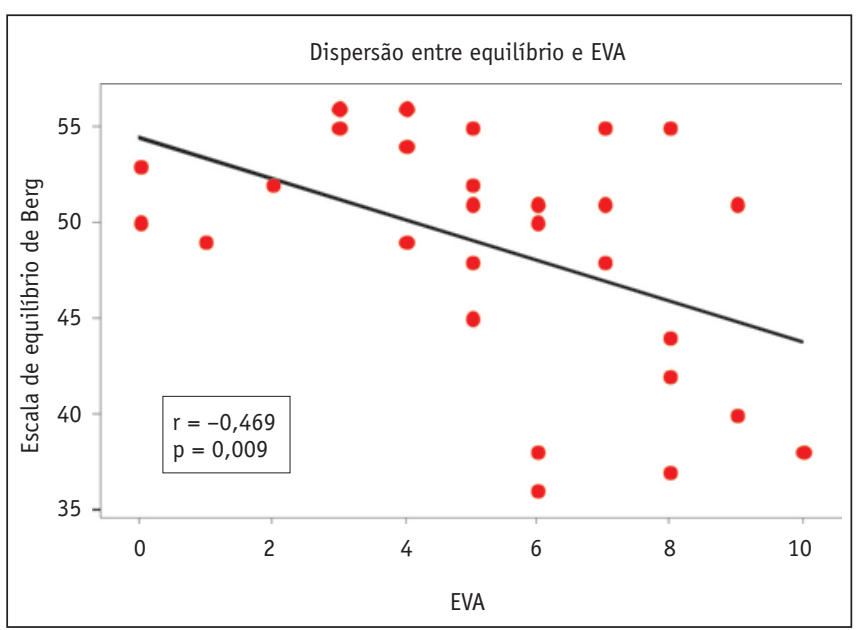

Figura 1 - Correlação entre escala de equilíbrio de Berg e dor nos pacientes com EA.

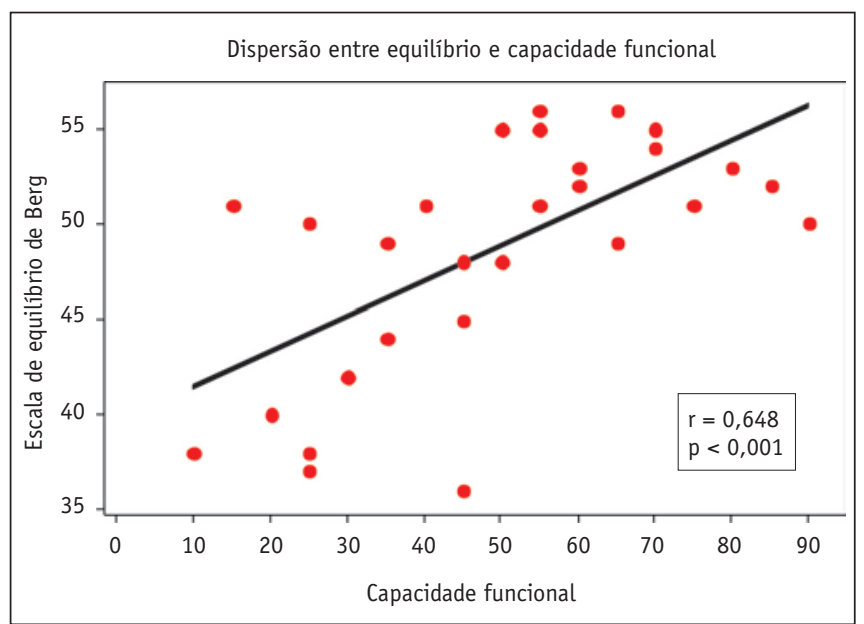

Figura 2 - Correlação entre escala de equilíbrio de Berg e capacidade funcional do SF-36 nos pacientes com espondilite anquilosante.

\section{DISCUSSÃO}

O objetivo foi avaliar o equilíbrio funcional em pacientes com o diagnóstico de EA, uma vez que essa é uma das alterações que pode ocorrer nesta doença.

De acordo com os resultados deste estudo, os pacientes com EA possuem pior equilíbrio quando avaliados pela escala de Berg, se comparados a indivíduos que não tenham EA.

A média do escore da escala de Berg dos pacientes estudados foi 48,83 , valores menores que 45 indicam comprometimento grave do equilíbrio. Apesar de os pacientes com EA não apresentarem resultado da escala de Berg menor que 45, quando comparados com indivíduos saudáveis apresentam pior equilíbrio. Acredita-se que os pacientes com EA tenham, ao longo da doença, se adaptado às alterações posturais e que o equilíbrio postural pode ser compensado por outras articulações não afetadas ${ }^{(5,16)}$. Uma compensação freqüente nesses pacientes é a rotação posterior da pelve, resultando grande aumento na angulação do tronco. No entanto, esta compensação pode ficar insuficiente por causa da progressão da doença, o que pode levar a permanente substituição do centro de massa no tronco, o que piora a "postura do esquiador". Mudanças posturais podem levar a pior equilíbrio em pacientes com EA, embora no estudo de Aydog et al. ${ }^{(5)}$ não tenha sido encontrado pior equilíbrio em EA avaliados pela plataforma de equilíbrio Biodex Stability System, diferindo dos resultados deste estudo. Talvez essa diferença entre os estudos seja por causa da utilização de diferentes instrumentos de avaliação do equilíbrio. O estudo de Murray et al. ${ }^{(7)}$ utilizou a magnetometria para avaliar o 
equilíbrio em pacientes com EA e concluiu que eles possuem pior equilíbrio do que pessoas saudáveis. Não foram encontrados, na literatura estudada, trabalhos que avaliassem o equilíbrio pela escala de Berg em pacientes com EA.

Encontra-se correlação positiva entre a pontuação da escala de equilíbrio de Berg e a pontuação da EVA $(\mathrm{r}=0,469) \mathrm{p}<0,001$, sugerindo que quanto maior a dor do paciente, pior seu equilíbrio funcional.

Quanto aos domínios do SF-36, encontra-se média correlação entre a capacidade funcional e a escala de Berg $(\mathrm{r}=0,649)$ e boa correlação da capacidade funcional com a $\operatorname{EVA}(\mathrm{r}=0,743)$, o que permite concluir que quanto pior a escala de Berg, pior será a capacidade funcional e dor.

No trabalho de Ozgul et al. ${ }^{(4)}$ sobre a qualidade de vida nos pacientes com EA, as piores pontuações do SF-36 foram obtidas nos domínios de limitação por aspectos físicos, estado geral de saúde e dor; o que também foi encontrado neste estudo, porquanto no trabalho citado não houve comparação com o grupo-controle.

\section{REFERÊNCIAS}

1. Carette S, Graham D, Little H, Rubenstein J, Rosen P: The natural disease course of ankylosing spondylitis. Arthritis Rheum 26(2): 186-90, 1983.

2. Viitanen JV, Heikkilã S: Functional changes in patients with spondylarthropathy. A controlled trial of the effects of short-term rehabilitation and 3-year follow-up. Rheumatol Int 20: 211-4, 2001.

3. Liu Y, Cortinovis D, Stone MA: Recent advances in treatment os the spondyloarthropathies. Curr Opin Rheumatol 16: 357-65, 2004.

4. Ozgul A, Peker F, Taskaynatan MA, Tan AK, Dinçer K, Kalyon TA: Effect of ankylosing spondylitis on health-related quality of life and different aspects of social life in young patients. Clin Rheumatol 25: 168-74, 2006.

5. Aydog E, Depedibi R, Bal A, Eksioglu E, Unlu E, Çakci A: Dynamic postural balance in ankylosing spondylitis patients. Rheumatology 45: 445-8, 2006.

6. Khan MA: Ankylosing spondylitis: clinical features. In: Klippel JH, Dieppe PA, editors. Rheumatology. St Louis: Mosby; 1994.

7. Murray HC, Elliot C, Barton SE, Murray A: Do patients with ankylosing have poorer balance than normal subjects? Rheumatology 39: 497-500, 2000.

8. Miyamoto ST, Lombardi Jr I, Berg KO, Ramos LR, Natour J: Brazilian version of the Berg balance scale. Braz J Med Biol Res 37: 1411-21, 2004.

9. Thorbahn LDB, Newton RA: Use of the Berg Balance Test to predict falls in elderly persons. Phys Ther 76: 576-85, 1996.

10. Shumway-Cook A, Baldwin M, Polissar NL, Gruber W: Predicting the probability for falls in community-dwelling older adults. Phys Ther 77: 812-9, 1997.
Turan et al. ${ }^{(17)}$, avaliando a qualidade de vida em 46 pacientes com EA, por meio do SF-36, encontrou valores baixos de limitações por aspectos físicos, dor, estado emocional, indicando que esses indivíduos também não possuíam boa qualidade de vida, o que também corrobora com os resultados deste estudo. No estudo de Ariza-Ariza, Hernandez-Cruz e Navarro-Sarabia ${ }^{(18)}$, encontrou-se piora maior na função física do que no estado emocional dos pacientes com EA, e Bostan et al. ${ }^{(19)}$, também encontrou pior qualidade de vida em pacientes com EA.

Não foi encontrada correlação entre o tempo de diagnóstico da doença e o equilíbrio.

\section{CONCLUSÃO}

Os pacientes com EA apresentam pior equilíbrio funcional, pior qualidade de vida e mais dor que indivíduos saudáveis. $\mathrm{O}$ pior equilíbrio correlacionou-se com pior capacidade funcional e dor.

11. Harada U, Chiu V, Damom-Rodriguez J, Fowler E, Siu A, Reuben DB: Screening for balance and mobility impairment in elderly individual living in residual care facilities. Phys Ther 75: 462-9, 1995.

12. Berg KO, Wood-Dauphinee S, Willians JI: The balance scale: reliability assessment with elderly residents and patients with an acute stroke. Scan J Rehab Med 27: 27-36, 1995.

13. Van der Linden S, Valkenburg HA, Cats A: Evaluation of diagnostic criteria for ankylosing spondylitis: a proposal for modification of the New York criteria. Arthritis Rheum 27(4): 361-8, 1984.

14. Ciconelli RM, Ferraz MB, Santos W, Meinao I, Quaresma MR: Tradução para a língua portuguesa e validação do questionário genérico de avaliação de qualidade de vida SF-36 (Brasil SF-36). Rev Bras Reumatol 39(3): 143-50, 1999.

15. Huskisson EC: Measurement of pain. Lancet 2: 1127-31, 1974.

16. Bot SDM, Caspers M, Van Royen BJ, Toussaint HM, Kingma I: Biomechanical analysis of posture in patients with spinal kyphosis due to ankylosing spondylitis: a pilot study. Rheumatol 38: 441-3, 1999.

17. Turan Y, Duruoz MT, Cerrahoglu L: Quality of life in patients with ankylosing spondylitis: a piloty study. Rheumatol Int Jan 25, 2007 [Epub ahead a print].

18. Ariza-Ariza R, Hernandez-Cruz B, Navarro-Sarabia F: Physical function and health - related quality os life of Spanish patients with ankylosing spondylitis. Arthritis Rheum 49(4): 483-7, 2003.

19. Bostan EE, Borman P, Bodur H, Barça N: Functional disability and quality of life in patients with ankylosing spondylitis. Rheumatol Int 23: 121-6, 2003. 